\title{
Inhalt
}

Professor Hans Willgerodt zum Gedächtnis

Hans Jörg Hennecke

Wertvolle Wissenschaft - Anmerkungen zu Hans Willgerodt, Werten und Wissen.

Beiträge zur Politischen Ökonomie

Andreas Freytag

Was ist ein politischer Ökonom? - Zum Beitrag von Hans Willgerodt zur Theorie der Wirtschaftspolitik

Hauptteil

Jesús Huerta de Soto

Die Verteidigung des Euro: ein österreichischer Ansatz

(Mit einer Kritik der Fehler der EZB und des Interventionismus aus Brüssel).

Alfred Schüller

Vom Euro zum Goldstandard?

Eine Replik auf den Beitrag von Jesús Huerta de Soto

Markus C. Kerber

Spielmacher der Wettbewerbsverfälschung?

Anmerkungen zur Rolle der EZB auf den Kapitalmärkten

Albrecht F. Michler und Markus Penatzer

Finanztransaktionssteuer: Zielsetzungen und potenzielle Auswirkungen

Xingyuan Feng, Christer Ljungwall und Sujian Guo

Re-Interpreting the "Chinese Miracle": A Multi-Dimensional Framework

Erich Weede

Ergänzende Anmerkungen zum chinesischen Wunder

Manuel Wörsdörfer und Carsten Dethlefs

Homo oeconomicus oder Homo culturalis? -

Aktuelle Herausforderungen für das ordoliberale Menschenbild

Ulrich Witt

Ordnungsökonomik und Soziale Marktwirtschaft in Bedrängnis

René Ruske und Johannes Suttner

Wie (un-)fair sind Ökonomen? -

Neue empirische Evidenz zur Marktbewertung und Rationalität 


\section{Michael Pickhardt}

Pareto meets Olson - A Note on Pareto-optimality and Group Size in Linear Public Goods Games.

Ludger Heidbrink

Unternehmen als politische Akteure. Eine Ortsbestimmung zwischen

Ordnungsverantwortung und Systemverantwortung.

Ingo Pies

Systemverantwortung versus Ordnungsverantwortung? -

Eine ordonomische Replik auf den Beitrag von Ludger Heidbrink

Andreas Suchanek

Unternehmensverantwortung als Vermeidung relevanter Inkonsistenzen

Bernd Noll

Unternehmen und beschäftigungspolitische Verantwortung -

eine historisch-genetische Annäherung.

Dominik H. Enste und Michael Hüther

Bürgerschaftliches Engagement der Unternehmen im öffentlichen Raum

Erik Gawel und Wolfgang Bretschneider

Recht auf Wasser - eine institutionenökonomische Perspektive.

Florian Drevs und Tristan Nguyen

Adverse Selektion light - Der Einfluss des Flat-Rate-Bias

auf das Tarifwahlverhalten bei Krankenversicherungen

Vorträge zur Ordnung der Wirtschaft und Gesellschaft

Erich Weede

Wahrheit und Gewissheit; Klimaschutz und Politik

Lars P. Feld

Europa in der Welt von heute:

Wilhelm Röpke und die Zukunft der Europäischen Währungsunion

Ernst-Joachim Mestmäcker

Wettbewerbsfreiheit und Wohlfahrt - Ein ideengeschichtlicher Beitrag zum Verhältnis von Ökonomie und Recht

Buchbesprechungen

Personenregister

Sachregister . 